\title{
Simvastatin Partially Prevents Bone Loss in Tail- suspended Rat:Bone Mineral Density and Histomorphometry Analysis
}

\author{
Faming Tian', Xinhao Fan², Lei Xing ${ }^{3}$, Zhiwei Lv $^{4 *}$ \\ ${ }^{1}$ Department of Medical Research Center, Hebei United University, Tangshan, China \\ ${ }^{2}$ Department of Stomatology, Kailuan Hospital, Tangshan, China \\ ${ }^{3}$ Department of Senile Disease, Affiliated Hospital of Hebei United University, Tangshan, China \\ ${ }^{4}$ Department of Anatomy, Hebei United University, Tangshan, China \\ 1tfm9911316@163.com, ${ }^{*}$ ncmclzw30@126.com
}

\begin{abstract}
Background: Skeletal unloading induces osteopenia in the loaded bones. Simvastatin, as one of the HMG-CoA reductase inhibitors for lowering lipids, has been demonstrated its potential benefit in bone formation, while no study reported whether simvastatin could prevent the bone loss in unloaded bone. Objective: We performed this study to assess the effect of simvastatin on bone mass and bone formation in tail-suspension rat. Methods: Tailsuspension rats were treated with or without simvastatin for 3 weeks, The right femurs were removed for the measurement of bone histomorphometry after bone mineral density(BMD) assessment by dual-energy X-ray absorptiometry. Results: The total bone mineral density(tBMD) and distal bone mineral density(dBMD) of tailsuspended rats were significantly lower than that of normal rat, while proximal bone mineral density(pBMD) of normal rats was significantly higher than tail-suspended rat, but no statistical difference was observed between normal and simvastatin-treatment rats. Similar to the BMD results, histomorphometric assessment for the trabeculae bone of distal femurs showed markedly higher BV/TV and lower trabecular $\mathrm{Tb}$. Sp in normal rats compared to those of tailsuspended rats, while both the bone formation and bone resorption parameters were markedly increased in tail-suspended rats with or without simvastatin treatment, simvastatin showed stimulation on osteoid formation comparing to normal and tail-suspended rats. Conclusion: simvastatin treatment could partially prevent tailsuspention-induced osteoporosis in unloaded limb.
\end{abstract}

Index Terms - Tail-suspension, Simvastatin, Bone histomorphometry, Bone mineral density, Bone formation

\section{Introduction}

Statin is a specific inhibitor of 3-hydroxy-3-methylglutaryl coenzyme A (HMG-CoA) reductase, rate-limiting enzyme of the cholesterol synthesis pathway. In a large screening, Mundy et al. identified statins, including simvastatin, among 30,000 investigated agents to increase the expression of Bone morphogenetic protein (BMP-2) [1]. BMP-2 is one of the most examined and potent growth factors targeting bone formation in vivo[2,3].Simvastatin is a chemical modification of lovastatin, which is obtained by the replacement of 2-methyl-butyryl side chain of lovastatin with a 2,2-dimethyl-butyryl group.

Skeletal unloading induces osteopenia in the loaded bones[4]. Tail-suspended models in rats simulate the conditions of lack of mechanical stress such as spaceflight for the loaded bones[5]. In such a model, the animal bears the whole weight by its forelimbs, and the movement of its hindlimbs is free without weight bearing.

Although relatively abundant information about simvastatin indicates its possible beneficial effect on bone[6,7], whether simvastatin could prevent the bone loss in tail-suspended rat is still unknown. Therefore, the present study was performed to establish the rat model of simulated unloading and investigate the effects of simvastatin on bone mass, microarchitecture and bone formation/resorption activities in tail-suspended rat.

\section{Methods}

Animal model Twenty-four 9-week-old male SpragueDawley rats(weight: 170 g-180 g, Vital River, Beijing, China ) were randomized into three groups of eight animals each: G1, control group without tail-suspention; G2, the tailsuspended group and treated with vehicle; G3, the tailsuspended group and administered daily with $20 \mathrm{mg} / \mathrm{kg}$ of simvastatin by gavage. Double labeling was performed with subcutaneous injections of tetracylcin 10 days before sacrifice and of calcein 4 days before sacrifice.

The experiment was lasted for three weeks, and all animals were sacrificed one day after the final simvastatin administration. The right femurs were removed for the measurement of bone histomorphometry after bone mineral density(BMD) assessment by dual-energy X-ray absorptiometry.

Measurement of bone mineral density The femur was divided into four equal regions, distal femur, proximal femur, midshaft femur (the two equal regions of diaphysis were considered). The bone mineral density (BMD) in the right femur was measured after sacrifice. The femur was placed in the antero-posterior position on the $\mathrm{x}$-ray table, and BMD was measured by dual energy x-ray absorptiometry (DEXA) (Norland- XR36, USA) using small animal scan mode.

Bone histomorphometry The distal half of the right femur were fixed with $70 \%$ ethanol, then cut in half crosssectionally with a low-speed metallurgical saw, and then were dehydrated in ascending grades of ethanol, defatted in dimethyl benzene, and embedded in methyl-methacrylate without decalcification. Undecalcified $5 \mu \mathrm{m}$ sections were 
prepared using a Jung-K microtome (Reichert-Jung, Heidelberg, Germany). The sections were stained in von Kossa and Giemsa, and the unstained sections were used to observe double labeling.

In our study, the following parameters were examined using a semi-automatic digitizing system for bone histomorphometry (Leica QWin, Leica, Germany), with final magnification of $\times 200$ : trabecular bone volume (bone volume/tissue volume, BV/TV), trabecular thickness (Tb.Th), trabecular number (Tb.N), trabecular separation (Tb.Sp), percent labeled perimeter (\%L.Pm), mineral apposition rate (MAR), bone formation rate (BFR/BS), osteoid surface (OS/BS), eroded surface (ES/BS), and osteoclast number (Oc.N). The areas measured were defined by rectangular regions in the secondary spongiosa more than $1 \mathrm{~mm}$ apart from the metaphyseal junction in the growth plate and $1 \mathrm{~mm}$ from endocortical surfaces.

Statistical ananlysis All values are expressed as the means \pm standard deviations. The SPSS 15.0 statistical ananlysis software was used for statistical ananlysis. PairedSample T test was used for the comparation of differences between the two groups, A P value $<0.05$ was considered to indicate statistical significance.

\section{Results}

Bone mineral density The total bone mineral density(tBMD) and distal bone mineral density(dBMD) of G1 were significantly higher than those of G2 and G3, while proximal bone mineral density(pBMD) of G1 was significantly higher than G2,but had no significant difference with G3, G3 was higher than but had no significant diference with G2. (table 1)

Bone histomorphometry Similar to the BMD results, histomorphometric assessment for the trabeculae bone of distal femurs showed markedly higher trabecular bone volume (bone volume/tissue volume, BV/TV) and lower trabecular separation $(\mathrm{Tb} . \mathrm{Sp})$ in normal rats compared to those of tailsuspended rats, while both bone formation and bone resorption parameters, including percent labeled perimeter (\%L.Pm, indicating mineralizing Surface per Bone Surface), mineral apposition rate (MAR), bone formation rate (BFR/BS), osteoid surface (OS/BS), as well as the bone resorption parameters of eroded surface $(\mathrm{Er} / \mathrm{Pm})$, and osteoclast number (Oc.N)were markedly increased in tail-suspended rats, simvastatin showed stimulation on osteoid formation (Tab.2)

Tab.1 Results of BMD indices of femur $(n=8, g / c m 2, x \pm s)$

\begin{tabular}{|c|c|c|c|}
\hline Group & $\mathrm{G} 1$ & $\mathrm{G} 2$ & $\mathrm{G} 3$ \\
\hline $\mathrm{tBMD}\left(\mathrm{g} / \mathrm{cm}^{2}\right)$ & $0.1043 \pm 0.0061$ & $0.0896 \pm 0.0071^{*}$ & $0.0932 \pm 0.0131^{*}$ \\
\hline $\mathrm{pBMD}\left(\mathrm{g} / \mathrm{cm}^{2}\right)$ & $0.1080 \pm 0.0093$ & $0.0938 \pm 0.0118^{*}$ & $0.0972 \pm 0.0180$ \\
\hline $\mathrm{dBMD}\left(\mathrm{g} / \mathrm{cm}^{2}\right)$ & $0.0982 \pm 0.0096$ & $0.0775 \pm 0.0085^{*}$ & $0.0831 \pm 0.0115^{*}$ \\
\hline
\end{tabular}

${ }^{*} p<0.05$, compared to group G1

Tab.2 Results of bone histomorphometry $(\mathrm{n}=8, \mathrm{x} \pm \pm \mathrm{s})$

\begin{tabular}{|l|l|l|l|}
\hline & G1 & G2 & G3 \\
\hline BV/TV $(\%)$ & $25.28 \pm 5.25$ & $12.45 \pm 4.08^{*}$ & $15.72 \pm 4.52^{*}$ \\
\hline Tb.Th $(\mu \mathrm{m})$ & $67.46 \pm 8.44$ & $59.52 \pm 16.73$ & $62.35 \pm 11.51$ \\
\hline Tb,N $(\# / \mathrm{mm})$ & $2.79 \pm 0.11$ & $2.51 \pm 0.33$ & $2.66 \pm 0.30$ \\
\hline Tb,Sp $(\mu \mathrm{m})$ & $385.46 \pm 731.03$ & $1094.25 \pm 5586.62^{*}$ & $953 \pm 4581.56^{*}$ \\
\hline Er.Pm $(\%)$ & $11.23 \pm 3.42$ & $22.72 \pm 11.72^{*}$ & $21.33 \pm 6.29^{*}$ \\
\hline BFR/BS $\left(\mu \mathrm{m} / \mathrm{d}^{*} 100\right)$ & $19.26 \pm 2.36$ & $29.14 \pm 3.27^{*}$ & $33.23 \pm 2.24^{*}$ \\
\hline MAR $(\mu \mathrm{m} / \mathrm{d})$ & $1.31 \pm 0.11$ & $1.37 \pm 0.14$ & $1.38 \pm 0.15$ \\
\hline O.Pm $(\%)$ & $25.35 \pm 5.42$ & $27.29 \pm 5.84$ & $44.93 \pm 14.05^{* \#}$ \\
\hline Oc.N $\left(\# / \mathrm{mm}{ }^{2}\right)$ & $0.09 \pm 0.05$ & $0.33 \pm 0.10^{*}$ & $0.40 \pm 0.15^{*}$ \\
\hline
\end{tabular}

${ }^{*} p<0.05$, compared to group $\mathrm{G} 1,{ }^{*} p<0.05$, compared to group G2

\section{Discussion}


The present study demonstrate that 4-weeks hind limb unloading by tail-suspension cause markedly bone loss and deterioration in microarchitecture of distal femur in rat, while to our surprise, those findings of our histomorphometric assessment also indicate that unloading with tail suspension increases both trabecular bone formation and trabecular bone resorption. Simvastatin treatment partially prevent the proximal femur bone loss, and imply an stimulation of the bone formation by markedly higher activity of osteoid formation.

We detect the bone mineral density in different part of rat femur, tail-suspension caused decrease bone mineral density in total and all regions in this study, indicating unloading results in a non-region specific bone loss, while simvastatin treatment only showed a positive effect on proximal femur, which maybe due to the better blood supply in this region. Regarding pharmacokinetics, statins are selectively targeted to liver. Because of their high lipophilicity and first-pass effect, the bioavailability of lipophilic statins is very low, at less than $5 \%$ [8-10]. Due to their liver specificity and very poor distribution to the periphery, the probability of statins reaching the bone micro-environment is also very low, which maybe the main reason for that we only find preventive effect of simvastatin on bone loss at the proximal femur.

The results of histomorphometric assessment in this study seems not consistent with the prevous study by Sakata[11], in which they reported that after 2 weeks tailsuspension, decreases trabecular bone formation and increases trabecular bone resorption were observed in the proximal tibiae in mice, reasons for the unfamiliar results in these two studies might mainly for the different suspension period, in the present study, the rat were tail-suspended for 3 weeks, which was longer than that in the previous study, the rat we choose in this study were still in the age with ability of long bone growth, therefore after a rapid bone loss, the decreased bone mass and increased activity of bone resorption were probably converged into a stimulation of bone formation, while this feedback was not observed in Sakata study for the short experimental period. A much earlier study indicate that after a rapid bone loss and maintain in low level, the bone mass gradually returns toward normal level for the subsequent 7 days[12].

\section{Acknowledgements}

This work was financially supported by the Hebei Natural Science Foundation (C2005000580)

\section{References}

[1] Mundy G, Garrett R, Harris S, Chan J, Chen D, Rossini G, Boyce B,Zhao M, and Gutierrez G, Stimulation of bone formation in vitro and in rodents by statins, Science .286(1999) 1946-1949.

[2] Wang EA, Rosen V, D'Alessandro JS, Bauduy M, Cordes P, Harada T, Israel DI, Hewick RM, Kerns KM and LaPan P, Recombinant human bone morphogenetic protein induces bone formation, Proc Natl Acad Sci USA .87(1990)2220-2224.

[3] Deng ZL, Sharff KA, Tang N, Song WX, Luo J, Luo X, Chen J, Bennett E, Reid R, Manning D, Xue A, Montag AG, Luu HH, Haydon RC and He TC.Regulation of osteogenic differentiation during skeletal development, Front Biosci. 13(2008)2001 -2021.

[4] Ju YI, Sone T, Ohnaru K, Choi HJ, Fukunaga M.Differential effects of jump versus running exercise on trabecular architecture during remobilization after suspension-induced osteopenia in growing rats, J Appl Physiol. 112(2012):766-772.

[5] Habold C, Momken I, Ouadi A, Bekaert V. Effect of prior treatment with resveratrol on density and structure of rat long bones under tailsuspension, Bone Miner Metab. 29(2011):15-22.

[6] Nichols R, Hopman WM, Morton AR, Harman GJ, Holden RM, Statins are associated with a reduced risk of bone fracture in hemodialysis (HD) patients, Hemodial Int. 12(2008)275-279.

[7] Skoglund B, Aspenberg P,Locally applied Simvastatin improves fracture healing in mice,BMC Musculoskelet Disord. 8(2007)98.

[8] Corsino A, Bellosta S, Baetta R, Fumagalli R, Paoletti R and Bernini F, New insights into the pharmacodynamic and pharmacokinetic properties of statins, Pharmacol Ther. 84(1999) 413-428.

[9] Reinoso RF, SanchezNavarro A, Garcia MJ and Prous JR, Preclinical pharmacokinetics of statins, Methods Fin Clin Pharmacol. 24(2002) 593613.

[10] William D and Feely J, Pharmacokinetic-pharmacodynamic drug interaction with $\mathrm{HMG}-\mathrm{CoA}$ reductase inhibitors, Clin Pharmacokinet. 41(2002) 343-370.

[11] Sakata T, Sakai A, Tsurukami H, Okimoto N, Okazaki Y, Ikeda S, Norimura T, Nakamura T.Trabecular bone turnover and bone marrow cell development in tail-suspended mice, J Bone Miner Res. 14(1999) 1596-604.

[12] Globus RK, Bikle DD, Morey Holton E. The temporal response of bone to unloading, Endocrinology. 118(1986)733-742. 